\title{
Modelo de volatilidad en un mercado financiero colombiano
}

\author{
Volatility model in a Colombian financial market
}

\author{
Christian Cortes Garcia \\ Alvaro Cangrejo Esquive \\ christian.cortes@usco.edu.co \\ alvaro.cangrejo@usco.edu.co
}

\section{Resumen}

En este trabajo se presenta una breve introduccón a los instrumentos estadísticos y modelos necesarios para explicar la volatilidad de los precios de activos, al seguir la metodología de series temporales e involucrar el efecto de heterocedasticidad condicional. Con estos lineamientos definidos, se modela la volatilidad de los retornos en los precios de cierre diarios de acciones de la empresa colombiana de Cementos Argos S.A al tomar como referencia los modelos ARCH, GARCH, TGARCH, IGARCH, EGARCH, APARCH y SVt-AR(1) con el fin de determinar la efectividad de los modelos por fuera de la muestra. El modelo que mejor explica la volatilidad condicional de los retornos es el $\operatorname{EGARCH}(1,1)$ y el modelo que mejor realiza pronóisticos de volatilidad es el SVt-AR(1).

Palabras clave: Volatilidad condicional, volatilidad estocastica, función de autocorrelación, retornos diarios, distribución GED, criterios de información bayesiano.

\begin{abstract}
In this paper we present a brief introduction to the statistical instruments and models necessary to explain the volatility of asset prices, following the time series methodology, involving the effect of conditional heteroscedasticity. With these defined guidelines, we model the volatility of the daily closing price returns of the shares of the Colombian company of Cementos Argos SA, taking as reference the ARCH, GARCH, TGARCH, IGARCH, EGARCH and APARCH models and incorporate back- Testing, in contrast to an SVt-AR (1) model, to determine the effectiveness of the models outside the sample. The model that best explains the conditional volatility of returns is the $\operatorname{EGARCH}(1,1)$ and the model that best performs volatility forecasts is $\mathrm{SV} t-\mathrm{AR}(1)$.
\end{abstract}

Keywords: Conditional volatility, stochastic volatility, autocorrelation function, daily returns, GED distribution, Bayesian information criteria.

\footnotetext{
${ }^{a}$ Docente. Departamento de matematica y estadistica, Universidad Surcolombiana

${ }^{\mathrm{b}}$ Docente. Departamento de matematica y estadistica, Universidad Surcolombiana
} 


\section{Introducción}

La compañía colombiana de Cementos Argos (2012) S.A. nació en Medellín - Colombia en el año 1934 e inició producción en el año 1936, dedicándose principalmente a la explotación, producción y comercialización de cemento, concreto y cualquier otro material a base de cemento, cal o arcilla.

según lo informado en Argos (2017), cementos Argos cuenta con $51 \%$ de participación en el mercado colombiano; es el cuarto productor de cemento más grande en Latinoamérica, el único productor de cemento blanco en Colombia, y es el cuarto productor más grande en Estados Unidos. Cuenta con 7 plantas de cemento en Colombia, 4 en Estados Unidos y 1 en Honduras; 9 moliendas de clínker ubicadas en Estados Unidos, Haití, Panamá, República Dominicana, Honduras, la Guayana Francesa y Surinam; y 32 puertos y terminales de recepción y empaque. Su capacidad instalada total es de 24 millones de toneladas de cemento al año.

Actualmente Argos tiene dos tipos de acciones, ordinaria y preferencial, que se transan localmente en la Bolsa de Valores De Colombia (2011), donde las acciones ordinarias en circulación están adquiridas en su mayoría por Grupo Argos S.A. $(53 \%)$ y fondos extranjeros $(17.8 \%)$.

Por lo general, las series temporales de alta frecuencia que se observan en estos tipos de mercados financieros no siguen una distribución normal, caracterizÁndose principalmente por ser asimétricas, leptocurticas, elevada persistencia en la volatilidad, correlaciones en los retornos al cuadrados, entre otros. De igual forma, una medida importante en las finanzas es el riesgo asociado con un activo y la volatilidad es una medida de incertidumbre, objeto de estudio por los inversionistas al momento de invertir.

Algunas investigaciones suponen que la varianza es determinista o constante a lo largo del tiempo, al fijar como estimador puntual la varianza muestral de los retornos del activo. Sin embargo, los hechos estilizados de los activos financieros, tales como acciones, bonos o tasas de cambio, sugieren que la volatilidad no es constante. Por lo tanto, asumir homoscedasticidad en el tiempo no es un supuesto muy realista.

Aunque la volatilidad de un activo esta bien definida, no es directamente observable en la práctica, pues se observan son los precios de un activo y sus derivados. El hecho de que la volatilidad no es directamente observable tiene varias implicaciones importantes en su estudio y modelización. Por lo tanto, para modelar las variaciones de series financieras y acercarse un poco más a la realidad, se han desarrollado modelos, tanto de volatilidad condicional como de volatilidad estocástica, como los ARCH, GARCH, IGARCH, EGARCH, TGARCH, APARCH, SV-AR(1) y SVt -AR(1), como lo ilustran Tsay (2014) y Kastner (2015), los cuales permiten ajustar la serie de retornos de cualquier activo con el fin de identificar la estructura condicional respecto al tiempo de cotización que se encuentra detrásde los retor- 
nos. La efectividad de los modelos de volatilidad condicional pueden observarse en los trabajos realizados por Franco (2007), González (1996), Awartani (2005) y de Jesús Gutiérrez (2013).

Para los inversionistas, el análisis de los retornos de un activo presenta un resumen completo al momento de invertir sin que la escala de medición de los precios influya. Por tal motivo, el objetivo de este trabajo es pronosticar la volatilidad de los retornos de precios de cierre diarios de una de las acciones más cotizadas en la bolsa de valores de Colombia, Cementos Argos S.A, durante el periodo 08/julio/2007 - 17/marzo/2017. Para ello, se usan los modelos de volatilidad condicional ARCH, GARCH, TGARCH, IGARCH, EGARCH y APARCH y seleccionar el modelo que mejor se ajusten al conjunto de datos en estudio a través del criterio de información bayesiana (BIC). Por último, se realiza un contraste con un modelo SVt-AR(1), para determinar su efectividad y pronósticos por fuera de la muestra.

\section{Preliminares}

según Tsay (2014), una serie de tiempo se define como un proceso estocástico, esto es, una colección de variables $X_{t}$ ordenadas en un tiempo $t$, la cual puede ser estacionaria, esto es, si su media y su varianza son constantes en el tiempo y si el valor de la covarianza entre dos periodos depende solamente de la distancia o rezago entre estos dos periodos de tiempo y no del tiempo en el cual se ha calculado la covarianza, o no estacionaria, generalmente conocida como caminata aleatoria, representada por

$$
X_{t}=X_{t-1}+\epsilon_{t}
$$

donde $\epsilon_{t} \sim \mathcal{N}(0,1)$ es el termino de error.

Para determinar si una serie $X_{t}$ es estacionaria se usa la prueba de Dickey - Fuller, la cual contrasta

$$
\begin{array}{ll}
H o: & \phi_{1}=1, \\
H a: & \phi_{1}<1,
\end{array}
$$

para el modelo

$$
X_{t}=\phi_{1} X_{t-1}+\epsilon_{t}
$$

Un estadístico de prueba conveniente es la relación $t$ de la estimación Dickey Fuller de $\phi_{1}$ bajo la hipótesis nula. Para la ecuación (1),

$$
\begin{aligned}
\phi_{1} & =\frac{\sum_{t=1}^{T} X_{t-1} X_{t}}{\sum_{t=1}^{T} X_{t-1}^{2}}, \\
\sigma_{\epsilon}^{2} & =\frac{\sum_{t=1}^{T}\left(X_{t}-\phi_{1} X_{t-1}\right)^{2}}{T-1},
\end{aligned}
$$

Comunicaciones en Estadística, diciembre 2018, Vol. 11, No. 2 
donde $X_{0}=0$ y $T$ es el tamaño de la muestra. El estadístico es:

$$
\mathrm{DF}=\frac{\phi_{1}-1}{\operatorname{std}\left(\phi_{1}\right)}=\frac{\sum_{t=1}^{T} X_{t-1} \epsilon_{t}}{\sigma_{\epsilon} \sqrt{\sum_{t=1}^{T} X_{t-1}^{2}}} .
$$

Por otro lado, en una serie de tiempo puede presentar que los valores que toma una variable en el tiempo no son independientes entre si, sino que un valor determinado depende de los valores anteriores.

La función de autocorrelación simple (ACF) mide la correlación entre dos variables separadas por $k$ periodos, definidas por la ecuación

$$
\rho_{j}=\operatorname{cor}\left(X_{j}, X_{j-k}\right)=\frac{\operatorname{cov}\left(X_{j}, X_{j-k}\right)}{\sqrt{\operatorname{Var}\left(X_{j}\right)} \sqrt{\operatorname{Var}\left(X_{j-k}\right)}} .
$$

donde

$$
\begin{array}{r}
\rho_{0}=1, \\
-1<\rho_{j}<1, \\
\rho_{j}=\rho_{-j} .
\end{array}
$$

La prueba Ljung-Box muestra en forma conjunta de que todos los coeficientes de autocorrelación son simultáneamente iguales a cero, esto es, son independientes. Para ello, se contrasta el siguiente planteamiento de hipótesis

$$
\begin{array}{ll}
H o: & \rho_{1}=\rho_{2}=\cdots=\rho_{k}=0 \\
H a: & \rho_{i} \neq 0 \text { para algún } i=1,2, \cdots, k
\end{array}
$$

y estadístico igual a

$$
Q(k)=T(T+2) \sum_{i=1}^{k}\left(\frac{\rho_{i}^{2}}{n-i}\right) \sim \chi_{k}^{2},
$$

con $T$ el tamaño de la muestra.

\section{Descripción de los modelos}

Dado el comportamiento de las series financieras, una de las principales preocupaciones de los inversionistas es tener un conocimiento aproximado del riesgo que presentan las inversiones que realizan. A partir de los precios diarios $X_{t}$ se puede calcular y modelar la rentabilidad como una variable aleatoria a la volatilidad de sus rentabilidades. Para medir el riesgo de mercado, se investiga el comportamiento de todas esas variables que influyen en la determinación de su rendimiento, o se considera la serie de retornos de los precios de mercado, que para valores pequeños de la rentabilidad precio, resulta ser una buena aproximación de la rentabilidad real, y permite la suma de las rentabilidades. Dichos retornos define la tasa de variación del activo, el cual se calcula al depender del tiempo de sus cotizaciones. 
Si los activos se cotizan para un cierto tiempo $t$ de forma continua, los retornos son de la forma

$$
R_{t}=\frac{X_{t}-X_{t-1}}{X_{t-1}}
$$

en cambio, si se cotizan para un tiempo $t$ de forma discreta, entonces

$$
R_{t}=\ln \left(\frac{X_{t}}{X_{t-1}}\right),
$$

donde, para ambos casos, $X_{t-1}$ es el precio del activo en el tiempo $t-1$ y $X_{t}$ es el precio en el tiempo $t$.

Sea $R_{t}$ el retorno de un activo en un tiempo $t$ que se comporta, generalmente, como una distribución leptocúrtica, asimétrica y un proceso estacionario. La idea central detrásdel estudio de la volatilidad es probar la heterocedasticidad condicional de la serie $R_{t}^{2}$.

según Tsay (2014), para realizar estos modelos, es importante considerar la esperanza y varianza condicional de $R_{t}$ dado $F_{t-1}$, son:

$$
\begin{aligned}
\mu_{t} & =\mathrm{E}\left(R_{t} \mid F_{t-1}\right), \\
\sigma_{t}^{2} & =\operatorname{Var}\left(R_{t} \mid F_{t-1}\right)=\mathrm{E}\left[\left(R_{t}-\mu_{t}\right)^{2} \mid F_{t-1}\right],
\end{aligned}
$$

donde $F_{t-1}$ es el conjunto de información disponible en el tiempo $t-1$ que, por lo general, expresa los retornos pasados.

Se asume que $\mu_{t}$ y $R_{t}$ siguen un modelo $\operatorname{ARMA}(p, q)$, donde

$$
\begin{aligned}
& R_{t}=\mu_{t}+a_{t}, \quad a_{t} \sim \operatorname{IID}(0,1) \\
& \mu_{t}=\phi_{0}+\sum_{i=1}^{p} \phi_{i} R_{t-i}+\sum_{j=1}^{q} \theta_{j} a_{t-j}
\end{aligned}
$$

$\operatorname{con}\left|\phi_{i}\right|<1$ y $\left|\theta_{j}\right|<1$.

De la ecuación anterior se puede deducir que

$$
\sigma_{t}^{2}=\operatorname{Var}\left(R_{t} \mid F_{t-1}\right)=\operatorname{Var}\left(a_{t} \mid F_{t-1}\right),
$$

donde $\sigma_{t}$ es la volatilidad. Los modelos condicionales heterosce dásticos dados en la siguiente sección se ocupan de la evolución de $\sigma_{t}^{2}$. La forma bajo que $\sigma_{t}^{2}$ evoluciona con el tiempo distingue un modelo de volatilidad de otro. Los modelos heterosce dásticos condicionales se clasifican en dos categorías generales. Los de la primera categoría utilizan una función exacta para gobernar la evolución de $\sigma_{t}^{2}$, mientras que los de la segunda categoría usan una ecuación estocástica para describir $\sigma_{t}^{2}$.

El modelo GARCH pertenece a la primera categoría, mientras que el modelo SV 
se encuentra en la segunda categoría. La expresión $a_{t}$ hace referencia a los shocks o las innovaciones de un retorno en el tiempo $t$. El modelo de $\mu_{t}$ en la ecuación (2) se denomina la ecuación media para $R_{t}$ y el modelo $\sigma_{t}^{2}$ la ecuación de volatilidad para $R_{t}$.

Para la construcción de modelos $\sigma_{t}^{2}$ se verifica la existencia de correlaciones significativa en la serie $R_{t}^{2}$ mediante la prueba Ljung-Box con estadístico $Q(k)$ a la serie $a_{t}^{2}$.

Luego de proponer un modelo en media condicional $\mu_{t} \mathrm{y}$ un modelo en varianza condicional o estocástica $\sigma_{t}^{2}$, se verifica los supuestos en sus residuales estandarizados:

$$
\tilde{a}_{t}=\frac{a_{t}}{\sigma_{t}}
$$

Las pruebas para comprobar los supuestos son las siguientes:

- Los estadísticos de Ljung-Box de $\tilde{a}_{t}$ para verificar la no existencia de autocorrelaciones significativas y validar la ecuación en media.

- Los estadísticos de Ljung-Box de $\left(\tilde{a}_{t}\right)^{2}$ para probar la validez de la ecuación de la volatilidad condicional o estocástica.

- La adecuada distribución de $\tilde{a}_{t}$, según sea el caso, planteada en los términos de error $\epsilon_{t}$ de los modelo de volatilidad condicional o estocástica.

Lo siguiente será describir varios modelos de volatilidad condicional y modelos de volatilidad estocástica, tomados de (Tsay, 2014) y Kastner, 2016a), respectivamente.

\subsection{Modelos de volatilidad condicional}

\section{Modelo ARCH}

Los modelos de heteroscedasticidad condicional autorregresivo, ver (Engle, 1982), conocidos como modelos ARCH, recoge los episodios de agrupamiento temporal de volatilidad que suele observarse en las series de rentabilidad de casi todo mercado financiero, del cual el shock en el retorno no está autocorrelacionado, pero síque es dependiente. De este modo, el modelo $\operatorname{ARCH}(m)$ es dado por:

$$
\begin{aligned}
R_{t} & =\mu_{t}+a_{t} \\
a_{t} & =\sigma_{t} \epsilon_{t}, \quad \epsilon_{t} \sim \operatorname{IID}(0,1) \\
\sigma_{t}^{2} & =\alpha_{0}+\sum_{i=1}^{m} \alpha_{i} a_{t-i}^{2}
\end{aligned}
$$

donde $\epsilon_{t}$ son variables aleatorias independientes e idénticamente distribuidas con media uno y varianza cero, $\alpha_{0}>0, \alpha_{i} \geq 0$ para todo $i>0 \mathrm{y}$, para cumplirse la 
condición de estacionariedad en media, la suma de todos los parámetros es menor que la unidad. Se puede suponer que $\epsilon_{t}$ sigue una distribución normal $\epsilon_{t} \sim \mathcal{N}(0,1)$, una distribución $t$-student estandarizada $\epsilon_{t} \sim t_{\nu}^{*}(0,1)$, o una distribución de errores generalizada $(\operatorname{GED}) \epsilon_{t} \sim \operatorname{GED}_{\kappa}(0,1)$, ver (Jiménez Moscoso, 2014).

\section{Modelo GARCH}

En muchos casos, el modelo ARCH que recoge la estructura de autocorrelación en varianza necesita de un elevado número de retardos. Para evitar que el alto número de coeficientes en términos autoregresivos, generalmente bastante relacionados, produzca una importante pérdida de precisión en su estimación, se ha propuesto una parametrización alternativa, restringida, dependiente de un número reducido de parámetros, ver (Bollerslev, 1986).

El modelo de heteroscedasticidad condicional autoregresivo generalizado, conocido como $\operatorname{GARCH}(m, s)$, es dado por:

$$
\begin{aligned}
R_{t} & =\mu_{t}+a_{t}, \\
a_{t} & =\sigma_{t} \epsilon_{t}, \quad \epsilon_{t} \sim \operatorname{IID}(0,1) \\
\sigma_{t}^{2} & =\alpha_{0}+\sum_{i=1}^{m} \alpha_{i} a_{t-i}^{2}+\sum_{j=1}^{s} \beta_{j} \sigma_{t-j}^{2},
\end{aligned}
$$

donde $\epsilon_{t}$ es una sucesión de variables idénticamente distribuidas con media cero y varianza uno, $\alpha_{0}>0, \alpha_{i} \geq 0, \beta_{i} \geq 0$ y $\sum_{i=1}^{\max (m, s)}\left(\alpha_{i}+\beta_{i}\right)<1$ para garantizar la condición de estacionariedad en media. En este caso, $\alpha_{i}=0$ si $i>m$ y $\beta_{j}=0$ si $j>s$. La última restricción en $\alpha_{i}+\beta_{j}$ implica que la varianza condicional de $a_{t}$ es finita, mientras que la varianza condicional $\sigma_{t}^{2}$ evoluciona con el tiempo.

\section{Modelo IGARCH}

Las siglas IGARCH hacen referencia a un modelo generalizado autorregresivo integrado de heterocedasticidad condicionada. El propósito de esta variante de modelo $\operatorname{GARCH}(m, s)$ es estimar de una forma conveniente el efecto de apalancamiento. Para lograrlo, el modelo supone que la varianza incondicional es finita:

$$
\sum_{i=1}^{m} \alpha_{i}+\sum_{j=1}^{s} \beta_{j}=1 .
$$

Por tanto, el modelo IGARCH(m,s) es dado por:

$$
\begin{aligned}
R_{t} & =\mu_{t}+a_{t} \\
a_{t} & =\sigma_{t} \epsilon_{t}, \quad \epsilon_{t} \sim \operatorname{IID}(0,1) \\
\sigma_{t}^{2} & =\alpha_{0}+\sum_{i=1}^{m} \alpha_{i} a_{t-i}^{2}+\sum_{j=1}^{s} \beta_{j} \sigma_{t-j}^{2} .
\end{aligned}
$$

Comunicaciones en Estadística, diciembre 2018, Vol. 11, No. 2 
Este nuevo modelo hace que el efecto de la varianza condicional no desaparezca nunca, a diferencia del modelos $\operatorname{GARCH}(m, s)$, transcurriendo múltiples periodos de tiempo hasta que la varianza alcance de nuevo el valor medio, ver Tsay (2014).

\section{Modelo EGARCH}

según Tsay (2014), el modelo EGARCH, o modelo exponencial generalizado autorregresivo y condicionalmente heterocedístico, acaba con las asimetrías en la estimación del efecto de los shocks, al implementar una función $g\left(\epsilon_{t}\right)$ de las innovaciones $\epsilon_{t}$ y en las que por tanto el valor de las innovaciones queda recogido por medio de la expresión $\left|\epsilon_{t}\right|-\mathrm{E}\left(\left|\epsilon_{t}\right|\right)$. Matemáticamente:

$$
g\left(\epsilon_{t}\right)=\theta \epsilon_{t}+\gamma\left[\left|\epsilon_{t}\right|-\mathrm{E}\left(\left|\epsilon_{t}\right|\right)\right]
$$

donde $\theta$ y $\gamma$ son constantes reales. Tanto $\epsilon_{t}$ como $\left|\epsilon_{t}\right|-\mathrm{E}\left(\left|\epsilon_{t}\right|\right)$ son variables iguales e idénticamente distribuidas de media cero y distribuciones continuas. Por lo tanto, $\mathrm{E}\left[g\left(\epsilon_{t}\right)\right]=0$. La asimetría de $g\left(\epsilon_{t}\right)$ se puede ver reescribióndolo como

$$
g\left(\epsilon_{t}\right)=\left\{\begin{array}{lll}
(\theta+\gamma) \epsilon_{t}-\gamma \mathrm{E}\left(\left|\epsilon_{t}\right|\right) & \text { si } \quad \epsilon_{t} \geq 0 \\
(\theta-\gamma) \epsilon_{t}-\gamma \mathrm{E}\left(\left|\epsilon_{t}\right|\right) & \text { si } \quad \epsilon_{t}<0
\end{array}\right.
$$

Un modelo $\operatorname{EGARCH}(m, s)$ puede escribirse como

$$
\begin{aligned}
R_{t} & =\mu_{t}+a_{t} \\
a_{t} & =\sigma_{t} \epsilon_{t}, \quad \epsilon_{t} \sim \operatorname{IID}(0,1) \\
\operatorname{Ln}\left(\sigma_{t}^{2}\right) & =\alpha_{0}+\frac{1+\beta_{1} B+\cdots+\beta_{s-1} B^{s-1}}{1-\alpha_{1} B-\cdots-\alpha_{m} B^{m}} g\left(\epsilon_{t-1}\right),
\end{aligned}
$$

donde $\alpha_{0}$ es una constante, $B$ es el operador retraso tal que $B g\left(\epsilon_{t}\right)=g\left(\epsilon_{t-1}\right)$ y $1+\beta_{1} B+\cdots+\beta_{s-1} B^{s-1}$ y $1-\alpha_{1} B-\cdots-\alpha_{m} B^{m}$ son polinomios con ceros fuera del círculo unitario y no tienen factores comunes, esto es, que los valores absolutos de los ceros son mayores que 1. La ecuación anterior utiliza la parametrización ARMA para describir la evolución de la varianza condicional de $a_{t}$.

Para facilitar la estimación, se puede utilizar una forma alternativa para el modelo $\operatorname{EGARCH}(m, s)$ :

$$
\operatorname{Ln}\left(\sigma_{t}^{2}\right)=\alpha_{0}+\sum_{i=1}^{m}\left(\alpha_{i} \frac{\left|a_{t-i}\right|}{\sigma_{t-i}}+\gamma_{i} \frac{a_{t-i}}{\sigma_{t-i}}\right)+\sum_{j=1}^{s} \beta_{j} \operatorname{Ln}\left(\sigma_{t-j}^{2}\right),
$$

donde el signo positivo de $a_{t-i}$ contribuye a $\alpha_{i}\left(1+\gamma_{i}\right)\left|\epsilon_{t}\right|$ de la log volatilidad, mientras que el signo negativo de $a_{t-i}$ contribuye a $\alpha_{i}\left(1-\gamma_{i}\right)\left|\epsilon_{t}\right|$, donde $\epsilon_{t}=\frac{a_{t-i}}{\sigma_{t-i}}$ y, el parámetro $\gamma_{i}$ significa el efecto de apalancamiento de $a_{t-i}$.

\section{Modelo TGARCH}

La idea del modelo TGARCH es dividir la distribución de los shocks en intervalos disjuntos, para luego aproximar una función lineal por tramos para la desviación 
estándar condicional o para la volatilidad condicional. Si sólo hay dos intervalos, la división es normalmente en un umbral identificado con el número cero, donde la influencia de los shocks positivos se identifica con valores por encima de cero y negativos por debajo de este valor. En este caso, el modelo $\operatorname{TGARCH}(m, s)$, ver Tsay (2014), se puede escribir como:

$$
\begin{aligned}
R_{t} & =\mu_{t}+a_{t} \\
a_{t} & =\sigma_{t} \epsilon_{t}, \quad \epsilon_{t} \sim \operatorname{IID}(0,1) \\
\sigma_{t}^{2} & =\alpha_{0}+\sum_{i=1}^{m}\left(\alpha_{i}+\gamma_{i} N_{t-i} a_{t-i}^{2}\right)+\sum_{j=1}^{s} \beta_{j} \sigma_{t-i}^{2}
\end{aligned}
$$

donde $N_{t-i}$ es un indicador para $a_{t-i}$ definido por:

$$
N_{t-i}=\left\{\begin{array}{lll}
1, & \text { si } & a_{t-i}<0 \\
0, & \text { si } & a_{t-i} \geq 0
\end{array}\right.
$$

y $\alpha_{i}, \gamma_{i}, \beta_{j}$ son parámetros no negativos que satisfacen condiciones similares a los modelos GARCH. A partir del modelo, un numero positivo $a_{t-i}$ contribuye $\alpha_{i} a_{t-i}^{2}$ a $\sigma_{t}^{2}$, mientras que un numero negativo $a_{t-i}$ tiene un mayor impacto $\left(\alpha_{i}+\gamma_{i}\right) a_{t-i}^{2}$ con $\gamma_{i}>0$.

\section{Modelo APARCH}

El modelo TGARCH pertenece a la clase de modelos de poder asimétrico autoregresivo condicional heteroscedastico, denominado $\operatorname{APARCH}(m, s)$, el cual, según ver Tsay (2014), se describe como

$$
\begin{aligned}
R_{t} & =\mu_{t}+a_{t} \\
a_{t} & =\sigma_{t} \epsilon_{t}, \quad \epsilon_{t} \sim \operatorname{IID}(0,1) \\
\sigma_{t}^{\delta} & =\alpha_{0}+\sum_{i=1}^{m} \alpha_{i}\left(\left|a_{t-i}\right|+\gamma_{i} a_{t-i}\right)^{\delta}+\sum_{j=1}^{s} \beta_{j} \sigma_{t-j}^{\delta}
\end{aligned}
$$

donde $\delta>0$ y los coeficientes $\alpha_{0}, \alpha_{i}, \gamma_{i}$ y $\beta_{j}$ satisfacen ciertas condiciones de regularidad para que la volatilidad sea positiva.

\section{Modelo de volatilidad estocástica}

Como lo referencia (Kastner, 2016a), los modelos de heteroscedasticidad condicional asumen que la volatilidad puede ser observada un paso adelante con la información que proporciona las variables aleatorias $\epsilon_{t}$. En estos modelos, las noticias o choques se incorporan únicamente a través de las innovaciones $\epsilon_{t}$, es decir que sólo existen perturbaciones sobre la ecuación de la media. De esta forma, la volatilidad se estima a través de la información pasada, tornándose predecible porque para cada momento $t$ ya se conoce la información en $t-1$.

Comunicaciones en Estadística, diciembre 2018, Vol. 11, No. 2 
Un modelo más realista para la volatilidad se puede basar en una ecuación de comportamiento que tiene un componente predecible que depende de la información pasada, como en los GARCH, y un componente de ruido inesperado. Este tipo de modelación corresponde al modelo SVt-AR(1).

\section{Modelo SVt-AR(1)}

El modelo de volatilidad estocástica autorregresivo o SVt-AR(1) es dado por

$$
\begin{aligned}
R_{t} & =\sigma_{t} \epsilon_{t}, \\
\sigma_{t}^{2} & =\exp \left\{\frac{h_{t}}{2}\right\}, \\
h_{t} & =\mu+\phi\left(h_{t-1}-\mu\right)+\eta_{t},
\end{aligned}
$$

donde $\mu \in \mathbb{R}, h_{t}$ sigue un proceso estacionario $\operatorname{AR}(1)$ con parámetro $|\phi|<1$.

Se asume que $\epsilon_{t} \sim t_{\nu}(0,1)$ y $\eta_{s} \sim \mathcal{N}\left(0, \sigma_{\eta}^{2}\right)$ son las perturbaciones o ruidos no esperados para los retornos y para la volatilidad, respectivamente, mutuamente independiente para todo $t$ y $s$.

Los vector de parámetros $\theta=\left(\mu, \phi, \sigma_{\eta}\right)$ pueden seguir distribuciones a priori de tal forma que se toma la mediana, como estimador robusto, de cada distribución como parámetro puntual del modelo.

El parámetro $\mu$ sigue una distribución normal a priori $\mathcal{N}(b, B)$. según Kastner (2016a), se puede tomar $b=0$ y $B \geq 100$, pero experiencias de diversos autores con datos empíricos, su elección exacta no suele ser muy importante.

Para el parámetro $\phi \in(-1,1)$, se elige $(\phi+1) \sim 2 \mathcal{B}\left(a_{0}, b_{0}\right)$, lo que implica que esta distribución se encuentra en el intervalo $(-1,1)$ y garantiza la estacionariedad del proceso de volatilidad autorregresiva. según Kastner (2016a), se puede tomar $a_{0}=5$ y $b_{0}=1.5$.

Para la volatilidad de la log varianza $\sigma_{\eta} \in \mathbb{R}^{+}$, se elige $\sigma_{\eta}^{2} \sim B \times \chi_{1}^{2}$, donde la elección del hiperparámetro $B$ resulta ser de menor importancia en aplicaciones emṕíricas, siempre y cuando no se fije demasiado pequeño. En algunas investigaciones, se puede tomar $B=1$.

Se asume que la distribución a priori del parámetro de grados de libertad $\nu$ sigue una distribución uniforme sobre el intervalo $(a, b)$, esto es, $\nu \sim \mathcal{U}(a, b)$. según Kastner (2015), se puede tomar $\nu \sim \mathcal{U}(2,100)$.

Comunicaciones en Estadística, diciembre 2018, Vol. 11, No. 2 
Tabla 1: Descriptivos acciones CEMARGOS.

\begin{tabular}{|l|l|l|}
\hline \multirow{2}{*}{ Medidas } & \multicolumn{2}{|c|}{ estadístico } \\
\cline { 2 - 3 } & Precio de cierre & Retornos \\
\hline \hline Media & 9861.631 & 0.0007299 \\
\hline Mediana & 9670 & 0 \\
\hline Varianza & 1878935 & 0.0002966789 \\
\hline Desv. estándar & 1370.742 & 0.01722437 \\
\hline IQR & 2170 & 0.01840002 \\
\hline Asimétrica & -0.1736806 & -0.1922218 \\
\hline Curtosis & -0.700694 & 2.148553 \\
\hline
\end{tabular}

Fuente: Elaboración propia a partir de los datos de CEMARGOS

\section{Presentación de la información}

La serie temporal $X_{t}$ a utilizar son los retornos diarios de las acciones CEMARGOS de la empresa colombiana de Cementos Argos S.A. Para el cálculo de esta serie de retornos, se utilizan los precios de cierre diarios de acciones en los que existe mercado en el periodo comprendido entre $08 /$ julio/2012 y el 17/marzo/2017, con un total de 1165 datos, obtenidos por la bolsa de valores (De Colombia, 2011).

En la Tabla 1, muestra que los precios de cierre no siguen una distribución normal, estando ligeramente sesgada a la izquierda y platicúrtica, con un coeficiente de asimetría de -0.1736806 y curtosis de $-0.700694<0$.

Por otro lado, en la Figura 1, se deduce que entre los años 2012, 2013 y 2014 hubo un incremento significativo en los precios de sus acciones y una fuerte caída en lo transcurrido del año 2015, conllevando a obtener precios por debajo de sus rangos intercuartilicos. En el año 2016 y lo transcurrido del año 2017, se aumentador dichos precios, pero con caída s aun significativas.

En la modelación de series temporales es importante identificar si el fenómeno presenta un proceso estacionario a lo largo de tiempo $1 \leq t \leq 1165$. Al aplicar la prueba de Dickey-Fuller, dado en la Sección 2, que contrasta

Ho: La serie $X_{t}$ sigue un proceso no estacionario,

$H a$ : La serie $X_{t}$ sigue un proceso estacionario,

se concluye que, con un nivel de significancia del $5 \%$, la serie de los precios no se comporta estadisticamente como un proceso estacionario, con una probabilidad de $4.543 \%$. Por tanto, es de total interés analizar los retornos de la serie dada por la 
Figura 1: Boxplot por año precio CEMARGOS.

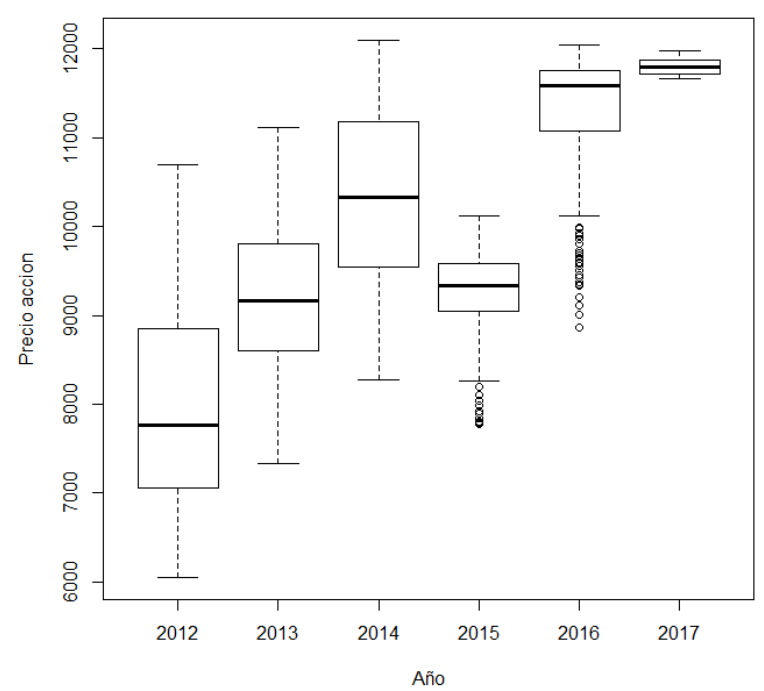

Fuente: Elaboración propia a partir de los datos de CEMARGOS

ecuación

$$
R_{t}=\frac{X_{t}-X_{t-1}}{X_{t-1}}
$$

donde $X_{t}$ es el precio de cierre en el tiempo $t$ y $X_{t-1}$ en el tiempo $t-1$.

De igual forma, al usar la prueba de Dickey-Fuller, el cual contrasta,

$$
H o \text { : La serie } R_{t} \text { sigue un proceso no estacionario, }
$$

$H a$ : La serie $R_{t}$ sigue un proceso estacionario,

se concluye que, con un nivel de significancia del $5 \%$, la serie se comporta estadisticamente como un proceso estacionario, con una probabilidad del $1 \%$.

En la Tabla 1 se observa que la serie $R_{t}$ no sigue una distribución normal, puesto que se encuentra ligeramente sesgada a la izquierda y leptocúrtica, con un coeficiente de asimetría de -0.1922218 y curtosis $2.148553>0$.

\section{4. análisis y resultados}

La serie de retornos $R_{t}$ en el periodo evaluado cuenta con 1164 datos. En la Figura 2 se observa la serie de los precios de cierre $\left\{X_{t}\right\}_{t=1}^{T=1165}$ y la serie de retornos 
Figura 2: Serie de precios y retornos CEMARGOS.

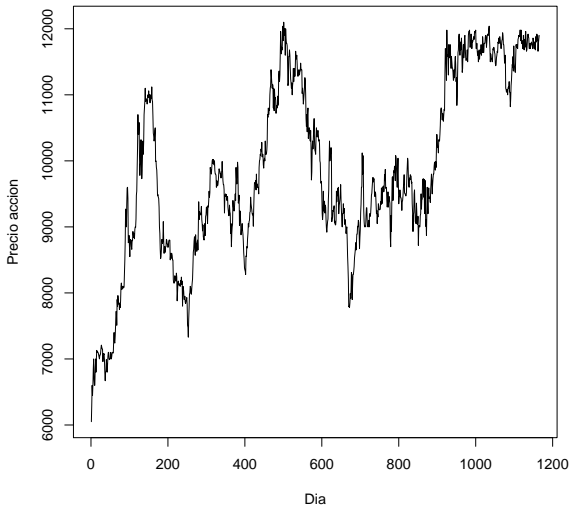

(a) Precios de cierre

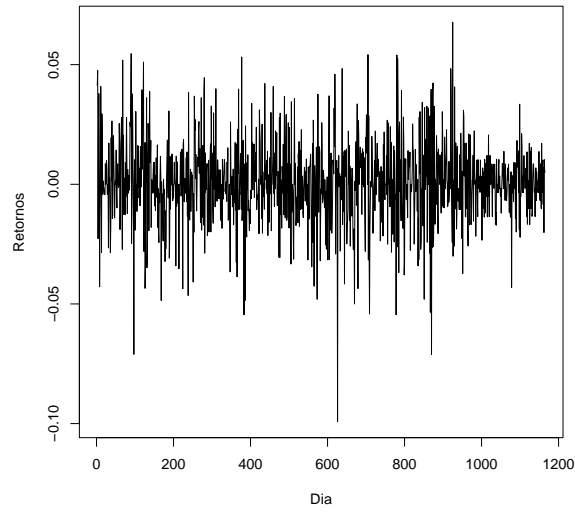

(b) Retornos

Fuente: Elaboración propia a partir de los datos de CEMARGOS

Figura 3: Histograma de acciones CEMARGOS.

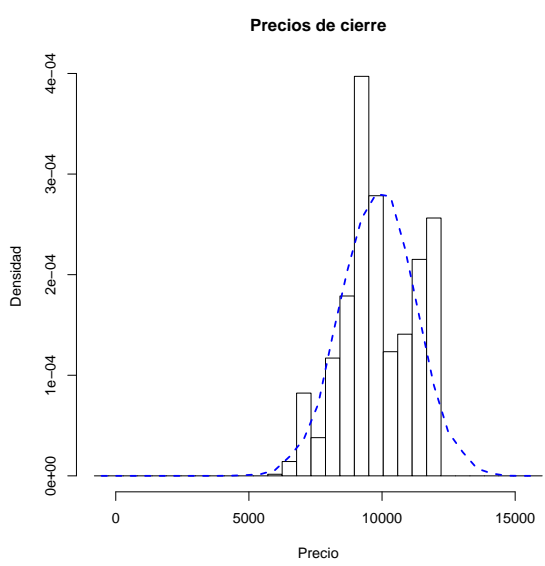

(a) Precios de cierre

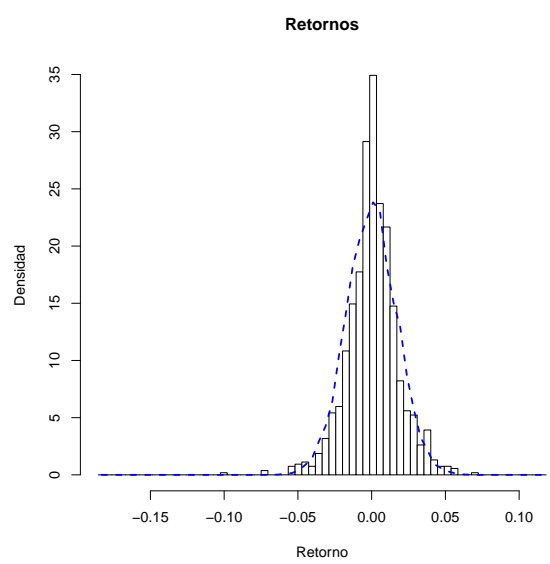

(b) Retornos

Fuente: Elaboración propia a partir de los datos de CEMARGOS

$\left\{R_{t}\right\}_{t=2}^{T=1165}$, el cual se evidencia fluctuaciones significativas.

El histograma de la Figura 3 muestra que la densidad incondicional de los retornos 
Figura 4: ACF retornos CEMARGOS.

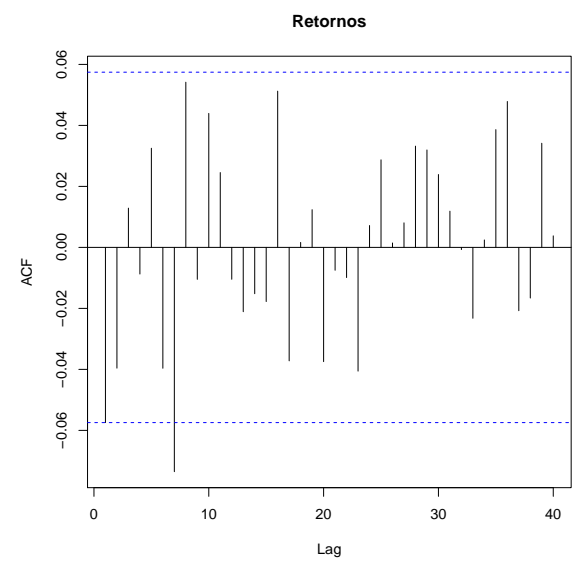

(a) $\mathrm{ACF}$ de $R_{t}$

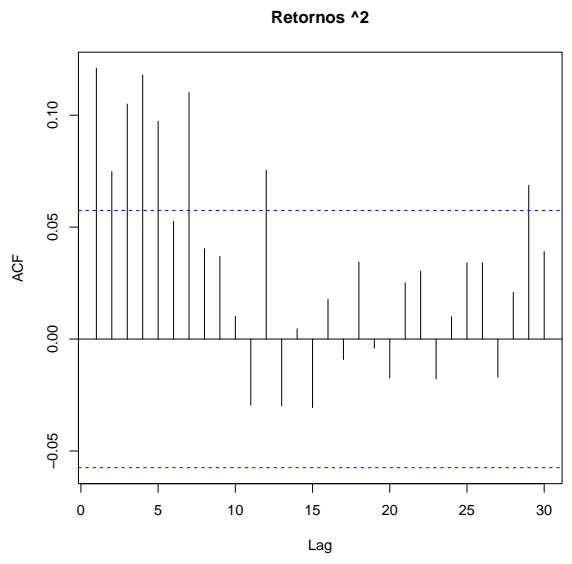

(b) $\mathrm{ACF}$ de $R_{t}^{2}$

Fuente: Elaboración propia a partir de los datos de CEMARGOS

es simétrica con un centramiento cercano a cero y se caracteriza por una curtosis mayor a la presentada en la distribución normal. Al aplicar una prueba $t$ para la serie de retornos, el cual contrasta

Ho: La media es igual a cero,

$H a$ : La media es diferente de cero,

evidentemente, con un nivel de significancia del $5 \%$, la media puede ser considerada estadisticamente como cero, con una probabilidad del $14.85 \%$.

En la Figura 4 se observa que la función de autocorrelación simple de la serie $R_{t}$ poseen pocos rezagos significativos que se encuentran por fuera de las bandas de confianza al $95 \%$, sin embargo se aplica la prueba de Ljung-Box para comprobar la significancia de la autocorrelaciones hasta el rezago 20. La probabilidad asociada al estadístico de prueba es del $7.259 \%$ el cual permite concluir, con un nivel de significancia del $5 \%$, que los retornos no presentan rezagos significativos. Se observan autocorrelaciones significativas en los retornos al cuadrado, con una probabilidad menor que 1\%, al aplicar prueba de Ljung-Box con nivel de significancia el $5 \%$, cual permite concluir que es necesario usar modelos para la volatilidad de los retornos.

A continuación se plantea los modelos de volatilidad condicional y estocásticas para la serie de retornos. 
Tabla 2: BIC modelos de volatilidad condicional para los retornos.

\begin{tabular}{|c|c|c|c|c|}
\hline & \multirow[t]{2}{*}{ Modelo } & \multicolumn{3}{|c|}{$\mathrm{BIC}$} \\
\hline & & $\epsilon_{t} \sim \mathcal{N}(0,1)$ & $\epsilon_{t} \sim t_{\nu}^{*}(0,1)$ & $\epsilon_{t} \sim \operatorname{GED}_{\kappa}(0,1)$ \\
\hline \multirow{9}{*}{$\mathrm{ARCH}$} & $\overline{\mathrm{ARCH}(1)}$ & -5.3190 & -5.3889 & 5.4003 \\
\hline & $\mathrm{ARCH}(2)$ & -5.3106 & -5.3815 & 5.3802 \\
\hline & $\mathrm{ARCH}(3)$ & -5.3086 & -5.3807 & 5.0021 \\
\hline & $\mathrm{ARCH}(4)$ & -5.3028 & -5.3752 & -5.3865 \\
\hline & $\mathrm{ARCH}(5)$ & -5.3046 & -5.3707 & -5.3827 \\
\hline & $\mathrm{ARCH}(6)$ & -5.3104 & -5.3681 & -5.3777 \\
\hline & $\mathrm{ARCH}(7)$ & -5.3167 & -5.3676 & -5.3769 \\
\hline & $\mathrm{ARCH}(8)$ & -5.3111 & -5.3619 & -5.3709 \\
\hline & $\operatorname{ARCH}(9)$ & -5.3058 & -5.3506 & -5.3659 \\
\hline \multirow{4}{*}{ GARCH } & $\operatorname{GARCH}(1,1)$ & -5.3522 & -5.4056 & -5.4167 \\
\hline & $\operatorname{GARCH}(1,2)$ & -5.3444 & -5.3989 & -5.4102 \\
\hline & $\operatorname{GARCH}(2,1)$ & -5.3438 & -5.3975 & -5.4089 \\
\hline & $\operatorname{GARCH}(2,2)$ & -5.3383 & -5.3928 & -5.4041 \\
\hline \multirow{4}{*}{ IGARCH } & IGARCH $(1,1)$ & -5.3438 & -5.4063 & -5.4159 \\
\hline & IGARCH $(1,2)$ & -5.3370 & -5.4000 & -5.4102 \\
\hline & IGARCH $(2,1)$ & -5.3358 & -5.3981 & -5.4084 \\
\hline & IGARCH $(2,2)$ & -5.3309 & -5.3939 & -5.4041 \\
\hline \multirow{4}{*}{ EGARCH } & EGARCH $(1,1)$ & -5.3511 & -5.4020 & -5.4133 \\
\hline & $\operatorname{EGARCH}(1,2)$ & -5.3435 & -5.3969 & -5.4072 \\
\hline & $\operatorname{EGARCH}(2,1)$ & -5.3408 & -5.3910 & -5.4027 \\
\hline & $\operatorname{EGARCH}(2,2)$ & -5.3361 & -5.3856 & -5.3975 \\
\hline \multirow{4}{*}{ TGARCH } & TGARCH $(1,1)$ & -5.3435 & -5.3983 & -5.4093 \\
\hline & TGARCH $(1,2)$ & -5.3319 & -5.3902 & -5.4011 \\
\hline & TGARCH $(2,1)$ & -5.3277 & -5.3826 & -5.3936 \\
\hline & TGARCH $(2,2)$ & -5.3198 & -5.3781 & -5.3890 \\
\hline \multirow{4}{*}{ APARCH } & $\operatorname{APARCH}(1,1)$ & -5.3414 & -5.4208 & -5.4055 \\
\hline & APARCH $(1,2)$ & -5.3311 & -5.3874 & -5.3984 \\
\hline & $\operatorname{APARCH}(2,1)$ & -5.3268 & -5.3785 & -5.3912 \\
\hline & $\operatorname{APARCH}(2,2)$ & -5.3246 & -5.3769 & -5.3888 \\
\hline
\end{tabular}

Fuente: Elaboración propia.

\subsection{Modelo de volatilidad condicional}

Con el fin de identificar un modelo apropiado, se inició con los modelos $\mathrm{ARCH}(m)$, $\operatorname{GARCH}(m, s), \operatorname{TGARCH}(m, s), \operatorname{EGARCH}(m, s), \operatorname{IGARCH}(m, s)$ y $\operatorname{APARCH}(m, s)$, con $\mu_{t}=0$ conforme al resultado de la prueba de Ljung-Box para la serie $R_{t}^{2}$, para valores de $m, s=1,2$ que son los primeros rezagos significativos observados en la ACP de la serie $R_{t}^{2}$. Como las series de retornos financieros poseen unas regularidades empíricas llamadas hechos estilizados, la cual se encuentran colas pesadas de 
la distribución de los retornos donde esta suele ser más leptocurtica que la normal, se propone el uso de la distribución $t$-student estandarizada y la distribución GED a las variables $\epsilon_{t}$.

En el Tabla 2 se muestran los valores dados por los criterios de información bayesiana BIC para cada uno de los modelos mencionados, respectivamente, por medio del paquete rugarch del programa estadístico R versión 3.3, ver (Ghalanos, 2013).

$\mathrm{Al}$ tomar los menores valores dados por el criterio de información bayesiano (BIC) para cada modelo, se selecciona el modelo $\mathrm{ARCH}(1) \operatorname{con} \epsilon \sim t_{4.4867}^{*}$ y los modelos $\operatorname{GARCH}(1,1), \operatorname{TGARCH}(1,1), \operatorname{EGARCH}(1,1), \operatorname{IGARCH}(1,1)$ y $\operatorname{APARCH}(1,1)$ con $\epsilon_{t} \sim \operatorname{GED}_{\kappa}(0,1)$. En las Tablas 3 y 4 se muestran los parámetros estimados con su nivel de significancia, y los estadísticos de prueba de Ljung-Box a los residuales estandarizados para cada uno de los modelos, respectivamente, de los cuales se concluye:

- El modelo ARCH(1) de la forma

$$
\begin{aligned}
R_{t} & =a_{t}, \\
a_{t} & =\sigma_{t} \epsilon_{t}, \quad \epsilon_{t} \sim t_{4.4867}^{*} \\
\sigma_{t}^{2} & =2.16 \times 10^{-3}+0.3858 a_{t-1}^{2},
\end{aligned}
$$

posee estadisticamente, para un nivel de significancia del $5 \%$, rezagos significativos en los residuales estandarizados al cuadrado como lo observado en la Tabla 4, por tanto no es un buen modelo que explique la volatilidad de la serie de retornos.

- El modelo IGARCH(1,1) de la forma

$$
\begin{aligned}
R_{t} & =a_{t}, \\
a_{t} & =\sigma_{t} \epsilon_{t}, \quad \epsilon_{t} \sim \operatorname{GED}_{1.109}(0,1) \\
\sigma_{t}^{2} & =9 \times 10^{-7}+0.161308 a_{t-1}^{2}+0.838692 \sigma_{t-1}^{2},
\end{aligned}
$$

los parámetros estimados son significativos, como lo observado en la Tabla 3 para un nivel de significancia del $5 \%$.

- El modelo $\operatorname{EGARCH}(1,1)$ dado por

$$
\begin{aligned}
R_{t}= & a_{t}, \\
a_{t}= & \sigma_{t} \epsilon_{t}, \quad \epsilon_{t} \sim \operatorname{GED}_{1.176}(0,1) \\
\operatorname{Ln}\left(\sigma_{t}^{2}\right)= & -0.84274+0.018381 \frac{\left|a_{t-1}\right|}{\sigma_{t-1}}+0.28686 \frac{a_{t-1}}{\sigma_{t-1}}+ \\
& +0.89754 \operatorname{Ln}\left(\sigma_{t-1}^{2}\right),
\end{aligned}
$$

el parámetro estimado $\alpha_{1}$ no es significativo, para un nivel de significancia del $5 \%$, con una probabilidad de $56.37 \%$. Al considerar $\alpha_{1}=0$, el nuevo modelo es expresado por:

$$
\begin{aligned}
R_{t} & =a_{t}, \\
a_{t} & =\sigma_{t} \epsilon_{t}, \quad \epsilon_{t} \sim \operatorname{GED}_{1.17598}(0,1) \\
\operatorname{Ln}\left(\sigma_{t}^{2}\right) & =-0.83994+0.28937 \frac{a_{t-1}}{\sigma_{t-1}}+0.89781 \operatorname{Ln}\left(\sigma_{t-1}^{2}\right),
\end{aligned}
$$


Tabla 3: Estimación de parámetros modelos de volatilidad condicional.

\begin{tabular}{|c|c|c|c|c|}
\hline Modelo & parámetro & Estimador & Error estándar & Probabilidad \\
\hline \multirow{3}{*}{$\mathrm{ARCH}(1)$} & $\alpha_{0}$ & 0.000216 & 0.0002 & $<0.01 \%$ \\
\hline & $\alpha_{1}$ & 0.385851 & 0.08196 & $<0.01 \%$ \\
\hline & $\nu$ & 4.486756 & 0.64196 & $<0.01 \%$ \\
\hline \multirow{4}{*}{$\operatorname{GARCH}(1,1)$} & $\alpha_{0}$ & 0.000028 & 0.000012 & $2.1047 \%$ \\
\hline & $\alpha_{1}$ & 0.157013 & 0.040552 & $<0.01 \%$ \\
\hline & $\beta_{1}$ & 0.757110 & 0.067094 & $<0.01 \%$ \\
\hline & $\kappa$ & 1.168351 & 0.072883 & $<0.01 \%$ \\
\hline \multirow{4}{*}{$\operatorname{IGARCH}(1,1)$} & $\alpha_{0}$ & 0.000009 & 0.000004 & $0.9011 \%$ \\
\hline & $\alpha_{1}$ & 0.161308 & 0.035461 & $<0.01 \%$ \\
\hline & $\beta_{1}$ & 0.838692 & - & - \\
\hline & $\kappa$ & 1.109824 & 0.067343 & $<0.01 \%$ \\
\hline \multirow{5}{*}{$\operatorname{EGARCH}(1,1)$} & $\alpha_{0}$ & -0.84274 & 0.301836 & $0.5238 \%$ \\
\hline & $\alpha_{1}$ & 0.01838 & 0.031855 & $56.3961 \%$ \\
\hline & $\beta_{1}$ & 0.89754 & 0.036735 & $<0.01 \%$ \\
\hline & $\gamma_{1}$ & 0.28686 & 0.055842 & $<0.01 \%$ \\
\hline & $\kappa$ & 1.17608 & 0.073525 & $<0.01 \%$ \\
\hline \multirow{5}{*}{$\operatorname{TGARCH}(1,1)$} & $\alpha_{0}$ & 0.002134 & 0.000794 & $0.7233 \%$ \\
\hline & $\alpha_{1}$ & 0.170026 & 0.035817 & $<0.01 \%$ \\
\hline & $\beta_{1}$ & 0.748807 & 0.065046 & $<0.01 \%$ \\
\hline & $\gamma_{1}$ & -0.054921 & 0.123345 & $65.6132 \%$ \\
\hline & $\kappa$ & 1.165914 & 0.072557 & $<0.01 \%$ \\
\hline \multirow{6}{*}{$\operatorname{APARCH}(1,1)$} & $\alpha_{0}$ & 0.000168 & 0.000334 & $61.3974 \%$ \\
\hline & $\alpha_{1}$ & 0.167804 & 0.042064 & $<0.01 \%$ \\
\hline & $\gamma_{1}$ & -0.055773 & 0.100267 & $57.8043 \%$ \\
\hline & $\beta_{1}$ & 0.755577 & 0.065200 & $<0.01 \%$ \\
\hline & $\delta$ & 1.585104 & 0.450267 & $0.0431 \%$ \\
\hline & $\kappa$ & 1.169885 & 0.072919 & $<0.01 \%$ \\
\hline
\end{tabular}

Fuente: Elaboración propia.

con $\mathrm{BIC}=-5.4190$.

- El modelo TGARCH(1,1) de la forma,

$$
\begin{aligned}
R_{t}= & a_{t}, \\
a_{t}= & \sigma_{t} \epsilon_{t}, \quad \epsilon_{t} \sim \operatorname{GED}_{1.165}(0,1) \\
\sigma_{t}^{2}= & 2.134 \times 10^{-3}+\left(0.170026-0.054921 N_{t-1}\right) a_{t-1}^{2}+ \\
& +0.748807 \sigma_{t-1}^{2},
\end{aligned}
$$

posee el parámetro $\gamma_{1}$ no significativo por ser un termino negativo, luego podría ser reducido a un modelo $\operatorname{GARCH}(1,1)$ dado por:

$$
\begin{aligned}
R_{t} & =a_{t}, \\
a_{t} & =\sigma_{t} \epsilon_{t}, \quad \epsilon_{t} \sim \operatorname{GED}_{1.1683}(0,1) \\
\sigma_{t}^{2} & =2.848 \times 10^{-5}+0.157013 a_{t-1}^{2}+0.75711 \sigma_{t-1}^{2} .
\end{aligned}
$$


Tabla 4: Probabilidades obtenidas por la prueba de Ljung-Box a residuales estandarizados.

\begin{tabular}{|l|l|l|l|l|l|l|}
\hline \multirow{2}{*}{ Modelo } & \multicolumn{3}{|c|}{$\tilde{a}_{t}$} & \multicolumn{3}{c|}{$\tilde{a}_{t}^{2}$} \\
\cline { 2 - 7 } & $Q(10)$ & $Q(15)$ & $Q(20)$ & $Q(10)$ & $Q(15)$ & $Q(20)$ \\
\hline \hline ARCH(1) & $16.14 \%$ & $36.17 \%$ & $24.54 \%$ & $0.04367 \%$ & $0.02827 \%$ & $0.01275 \%$ \\
\hline GARCH(1,1) & $16.65 \%$ & $37.15 \%$ & $29.44 \%$ & $95.81 \%$ & $35.66 \%$ & $42.9 \%$ \\
\hline IGARCH(1,1) & $15.76 \%$ & $37.39 \%$ & $28.54 \%$ & $98.22 \%$ & $50.41 \%$ & $45.69 \%$ \\
\hline EGARCH(1,1) & $15.5 \%$ & $33.63 \%$ & $27.57 \%$ & $98.75 \%$ & $29.45 \%$ & $37.51 \%$ \\
\hline TGARCH(1,1) & $17.82 \%$ & $36.55 \%$ & $31.33 \%$ & $96.12 \%$ & $20.3 \%$ & $29.97 \%$ \\
\hline APARCH(1,1) & $17.32 \%$ & $36.95 \%$ & $52.86 \%$ & $30.17 \%$ & $26.5 \%$ & $34.74 \%$ \\
\hline
\end{tabular}

Fuente: Elaboración propia.

- El modelo $\operatorname{APARCH}(1,1)$ formulado por:

$$
\begin{aligned}
R_{t} & =a_{t}, \\
a_{t} & =\sigma_{t} \epsilon_{t}, \epsilon_{t} \sim \operatorname{GED}_{1.169}(0,1) \\
\sigma_{t}^{1.23} & =7.291 \times 10^{-4}+0.169\left(\left|a_{t-1}\right|-0.063 a_{t-1}\right)^{1.23}+0.75 \sigma_{t-1}^{1.23}
\end{aligned}
$$

posee un parámetro negativo, no acorde a las condiciones básicas del modelo.

En base a los supuestos del modelo, la significancia de los parámetros, el menor BIC y las correlaciones nulas en los residuales estandarizados de los modelos mediante la prueba de Ljung-Box, el modelo que mejor se ajusta para explicar la volatilidad condicional de los retornos es un $\operatorname{EGARCH}(1,1)$ con $\alpha_{1}=0$, especificado en la ecuación (4).

Como lo observado en la Figura 5, la función de autocorrelación parcial de los residuales estandarizados al cuadrado poseen pocos rezagos significativos, por lo tanto el modelo de heterocedasticidad condicional está bien especificado; sin embargo se aplicó la prueba de Ljung-Box para verificar este resultado.

La probabilidad resultante es del $39.98 \%$ el cual indica, a un nivel de significancia del $5 \%$, que las autocorrelaciones conjuntas hasta el rezago 20 de los residuales estandarizados al cuadrado no son significativas. Esto indica que el modelo en varianza se encuentra adecuadamente especificado.

Asímismo, no se considera un modelo en media condicional $\mu_{t}$ ya que no existen correlaciones significativas con respecto a los residuales estandarizados, sin embargo se procedió aplicar la prueba Ljung-Box para evaluar formalmente la significancia de dichas correlaciones hasta el rezago 20.

La probabilidad resultante es del $19.66 \%$ el cual indica que no existe suficiente evidencia para rechazar la hipótesis nula y por lo tanto, las autocorrelaciones conjuntas hasta el rezago 20 de los residuales estandarizados no son significativas e 
Figura 5: ACF residuales estandarizados $\operatorname{EGARCH}(1,1)$.

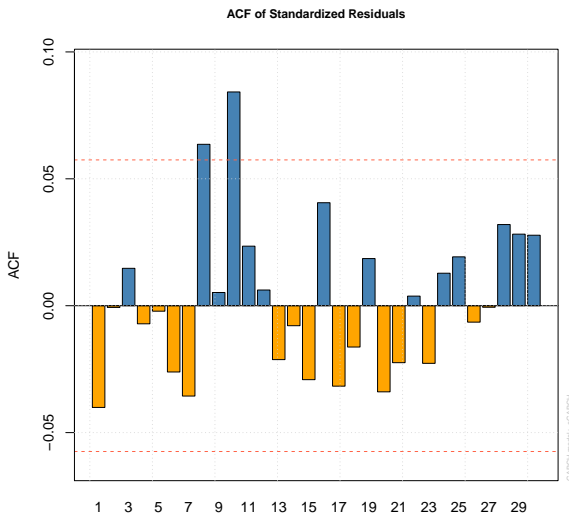

lag

(a) $\mathrm{ACF}$ de $\tilde{a}_{t}$

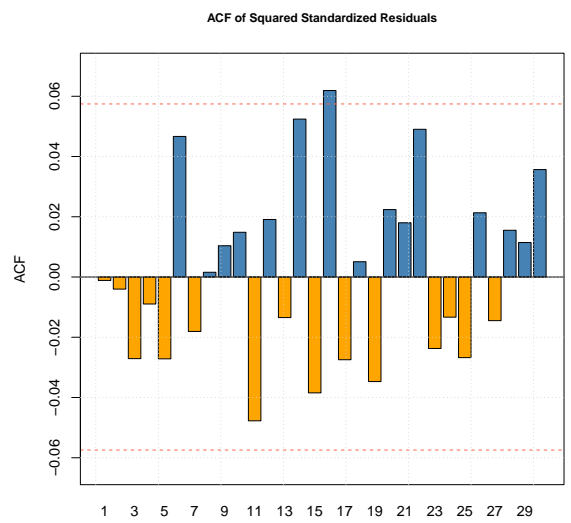

(b) $\mathrm{ACF}$ de $\tilde{a}_{t}^{2}$

Fuente: Elaboración propia.

Figura 6: Modelo EGARCH(1,1)

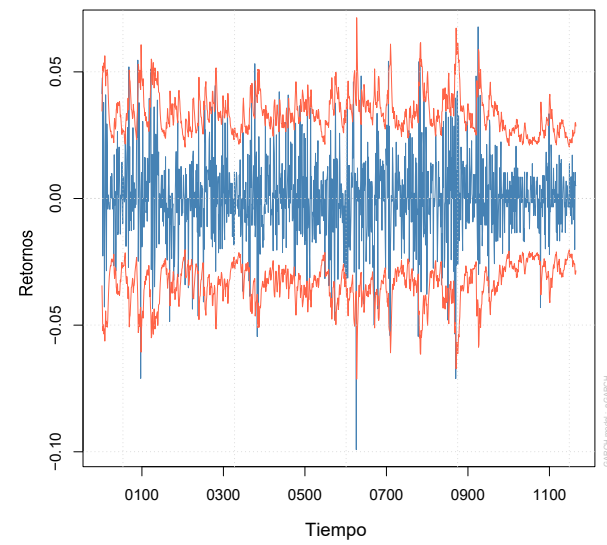

(a) Bandas de confianza al $95 \%$ para la volatilidad de los retornos

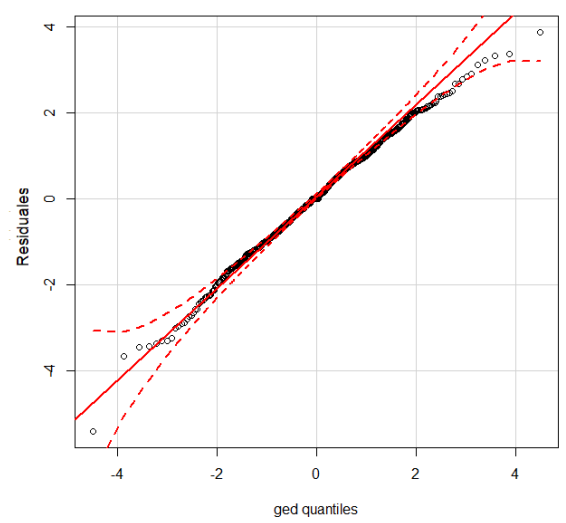

(b) Distribucion GED Residuales estandarizados

Fuente: Elaboración propia.

indica que el modelo en media condicional dado por $\mu_{t}=0$ se encuentra correctamente especificado.

Comunicaciones en Estadística, diciembre 2018, Vol. 11, No. 2 
Tabla 5: Estimaciones modelo SVt-AR(1).

\begin{tabular}{|l|l|l|l|l|l|}
\hline parámetro & media & desviación estándar & $5 \%$ & $50 \%$ & $95 \%$ \\
\hline \hline$\mu$ & -8.527 & 0.11 & -8.717 & -8.526 & -8.341 \\
\hline$\phi$ & 0.854 & 0.046 & 0.769 & 0.860 & 0.920 \\
\hline$\sigma_{\eta}$ & 0.447 & 0.082 & 0.320 & 0.441 & 0.589 \\
\hline$\nu$ & 46.675 & 25 & 13.434 & 41.799 & 91.654 \\
\hline $\exp (\mu / 2)$ & 0.014 & 0.00081 & 0.013 & 0.014 & 0.015 \\
\hline$\sigma_{\eta}^{2}$ & 0.207 & 0.076 & 0.103 & 0.194 & 0.347 \\
\hline
\end{tabular}

Fuente: Elaboración propia.

La Figura 6(a) muestra que el modelo en heterocedasticidad condicional logra capturar la volatilidad de los retornos a dos desviaciones estándar, con un nivel de confianza del $95 \%$ y confirma un buen ajuste del modelo.

Por otro lado, la verificación de la distribución de los errores (GED) con parámetro $\kappa=1.17$ se realizó de manera gráfica y se presentan en la Figura 6(b) se observa que los residuales estandarizados se ajustan correctamente a dicha distribución. Al aplicar la prueba de bondad de ajuste de Kolmogorov-Smirnov, no existe evidencia suficiente, a un nivel de significancia del $5 \%$, para determinar que los residuales estandarizados no siguen una distribucion GED, con una probabilidad del $32.8 \%$. Por tanto, los residuales estandarizados siguen una distribución GED.

\subsection{Modelo de volatilidad estocástica}

Mediante el paquete stochvol del software estadístico R, ver (Kastner, 2016b), se estiman los parámetros del modelo SVt-AR(1) de la serie $R_{t}$ donde $\mu \sim \overline{\mathcal{N}}(0,100)$, $\frac{\phi+1}{2} \sim \mathcal{B}(5,1.5), \sigma^{2} \sim \chi_{1}^{2} \mathrm{y} \nu \sim \mathcal{U}(2,100)$. Las estimaciones puntuales de dichos parámetros se muestra en la Tabla 5 .

Los residuales y las bandas de confianza al $95 \%$ para la volatilidad de los retornos son dadas en la Figura 7, cuyos parámetros fueron estimados a partir de la mediana para cada distribución a priori. Se observan que los residuales del modelo no sigue una distribución $t$-student, pues no todos los puntos caen en las bandas de confianza del $95 \%$, por tanto no es un buen modelo que logre estimar apropiadamente la volatilidad de los retornos.

\section{Modelo de pronóstico}

Al tomar los modelos $\operatorname{GARCH}(1,1)$, IGARCH(1,1) y $\operatorname{EGARCH}(1,1)$ dados por las ecuaciones (5), (3) y (4), respectivamente, se analiza la efectividad de los modelos 
Figura 7: Modelo SVt-AR(1).

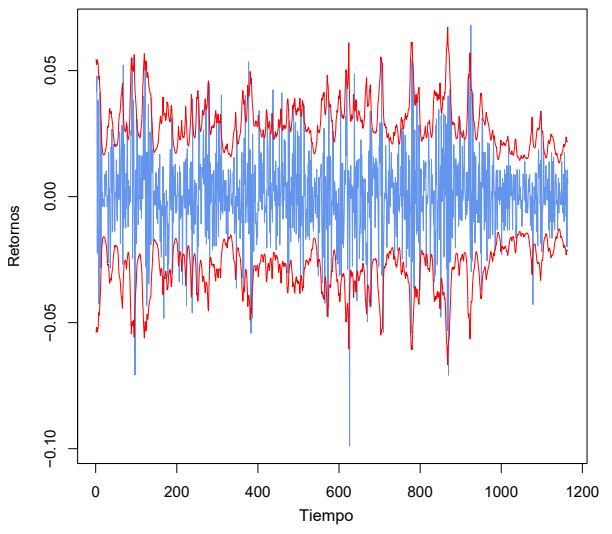

(a) Bandas de confianza al $95 \%$ para la volatilidad de los retornos

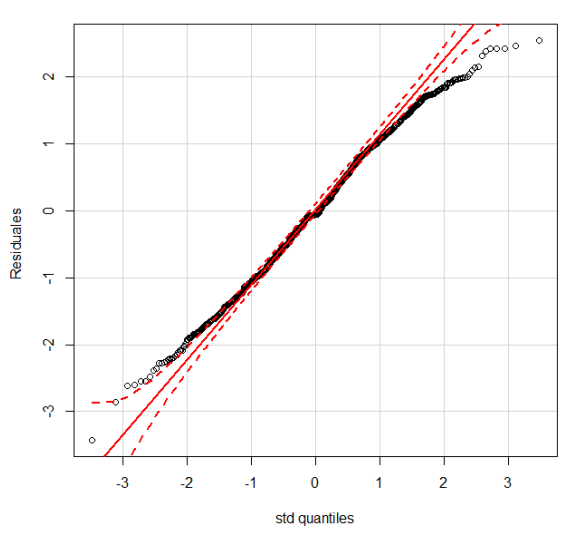

(b) Residuales estandarizados

Fuente: Elaboración propia.

al eliminar los últimos veinte datos de la serie de retornos $R_{t}$ y pronosticar sus volatilidades.

En la Figura 8 se observa que los últimos veinte datos de la serie de retornos $R_{t}$ se encuentran dentro de las bandas de confianza al $95 \%$ para la volatilidad pronosticadas por cada uno de los modelos tratados, por tanto los modelos GARCH(1,1), $\operatorname{EGARCH}(1,1)$ e $\operatorname{IGARCH}(1,1)$ pronostican adecuadamente las volatilidades de los últimos veinte datos de la serie de retornos $R_{t}$.

Por otro lado, al tomar como referencia los precios de cierre comprendidos entre el 21/03/2017 hasta 10/04/2017 obtenidos por la bolsa de valores de Colombia, se analiza si los modelos de volatilidad condicional $\operatorname{GARCH}(1,1), \operatorname{IGARCH}(1,1)$ y $\operatorname{EGARCH}(1,1)$ con el modelo de volatilidad estocástica SVt-AR(1) realizan un buen pronóstico de las volatilidades de la serie de retornos.

En la Figura 9 se observa que las bandas de confianza para cada modelo, con un nivel de confianza del $95 \%$, abarcan completamente los retornos comprendidos entre 21/03/2017 hasta 10/04/2017, esto es, los 15 pronósticos que se encuentran después de la línea $t=1165$. Sin embargo, dado que las volatilidades pronósticos para cada uno de los modelos va en forma creciente, el modelo SVt-AR(1) posee menos incertidumbre con relación a los modelos $\operatorname{IGARCH}(1,1), \operatorname{EGARCH}(1,1)$ y $\operatorname{GARCH}(1,1)$, pues sus volatilidades pronosticadas son inferiores al resto de los modelos de volatilidad condicional, conforme a lo mostrado en la Figura 10. 
Figura 8: Pronóstico serie de retornos recortada con los últimos 20 datos

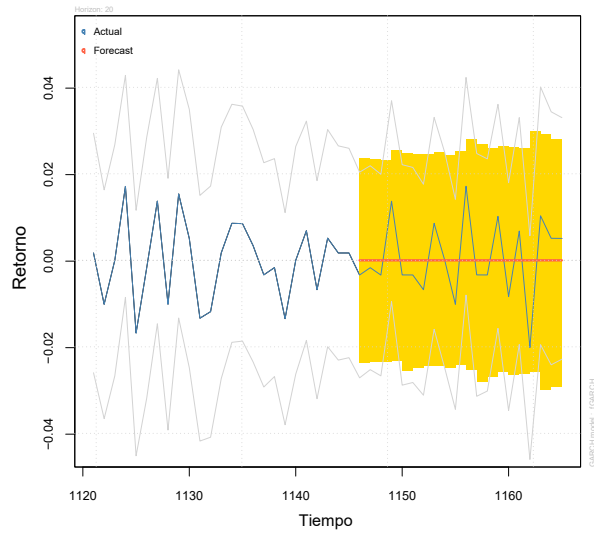

(a) $\operatorname{GARCH}(1,1)$

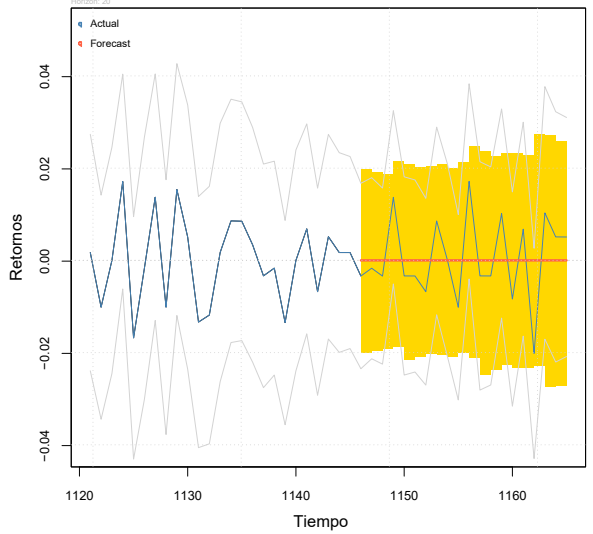

(b) $\operatorname{IGARCH}(1,1)$

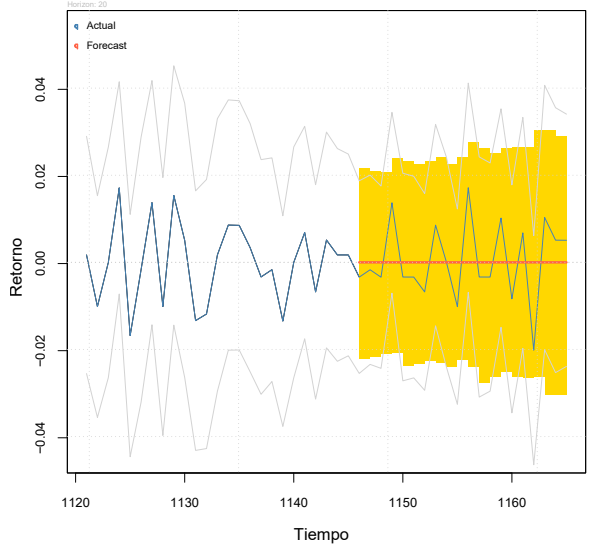

(c) $\operatorname{EGARCH}(1,1)$

Fuente: Elaboración propia.

En la Tabla 6 se muestran los primeros quince pronósticos, por medio de los modelos de volatilidad considerados, para los precios de cierre de acciones CEMARGOS entre el 21/03/2017 a 10/04/2017, donde los diversos de errores para dichos pronósticos, con $X_{t}$ los precios de cierre reales y $\widehat{X_{t}}$ los pronosticados, son dados 
Figura 9: Bandas de confianza al $95 \%$ para la volatilidad de los retornos $R_{t}$ con 15 datos posteriores a la línea $t=1165$.

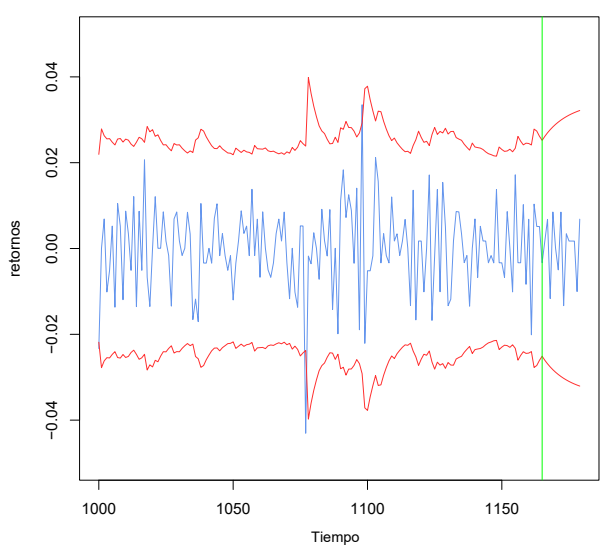

(a) $\operatorname{GARCH}(1,1)$

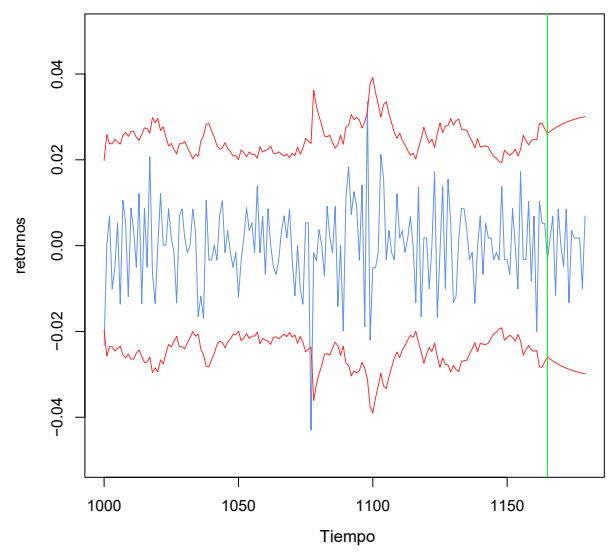

(c) $\operatorname{EGARCH}(1,1)$

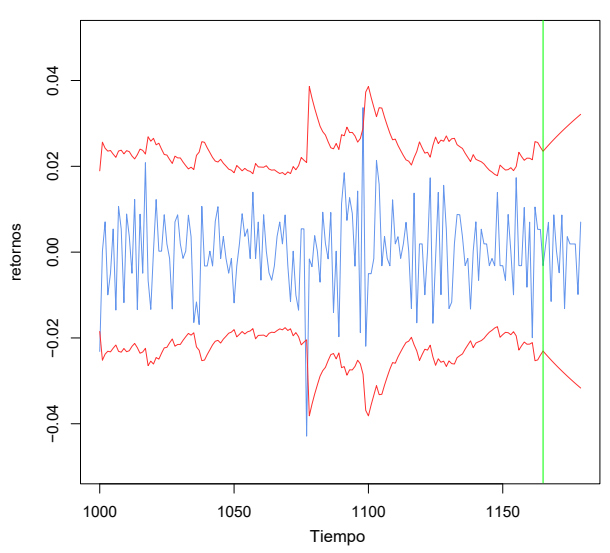

(b) $\operatorname{IGARCH}(1,1)$

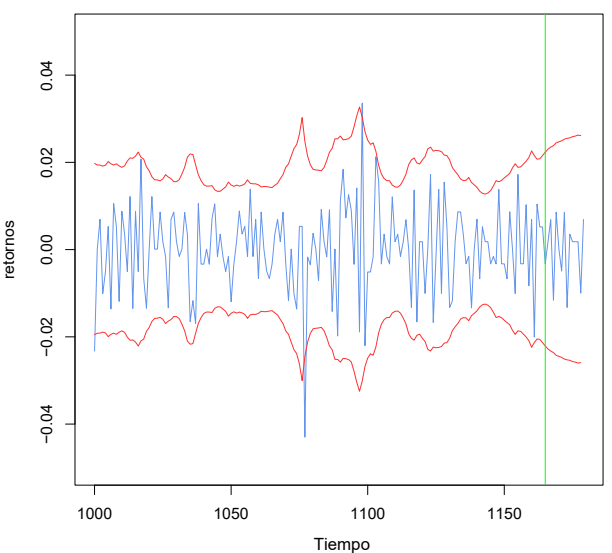

(d) SVt-AR(1)

Fuente: Elaboración propia.

por:

$$
\begin{aligned}
\mathrm{MAE} & =\frac{1}{15} \sum_{t=1166}^{1181}\left|X_{t}-\widehat{X}_{t}\right| \\
\mathrm{CME} & =\frac{1}{15} \sum_{t=1166}^{1181}\left(X_{t}-\widehat{X}_{t}\right)^{2},
\end{aligned}
$$


Figura 10: Pronóstico volatilidad de la serie $R_{t}$ para 15 datos.

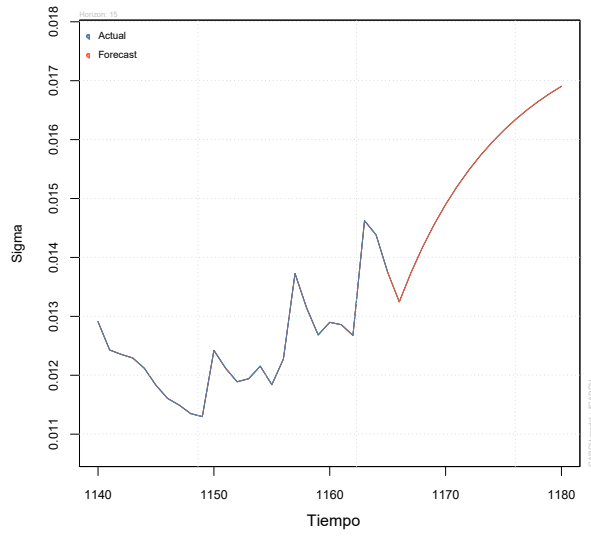

(a) $\operatorname{GARCH}(1,1)$

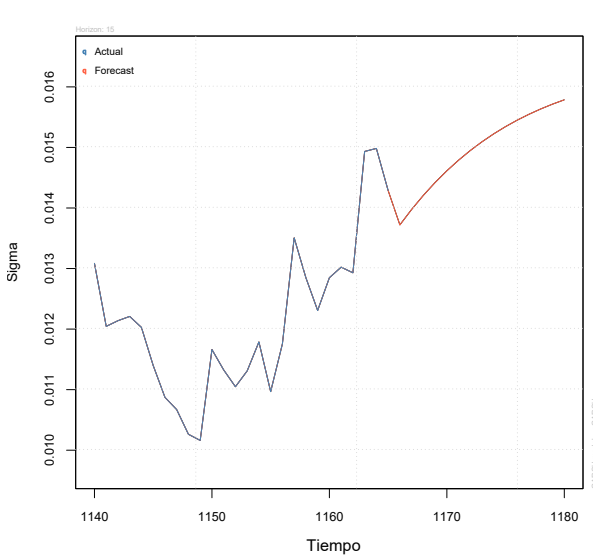

(c) $\operatorname{EGARCH}(1,1)$

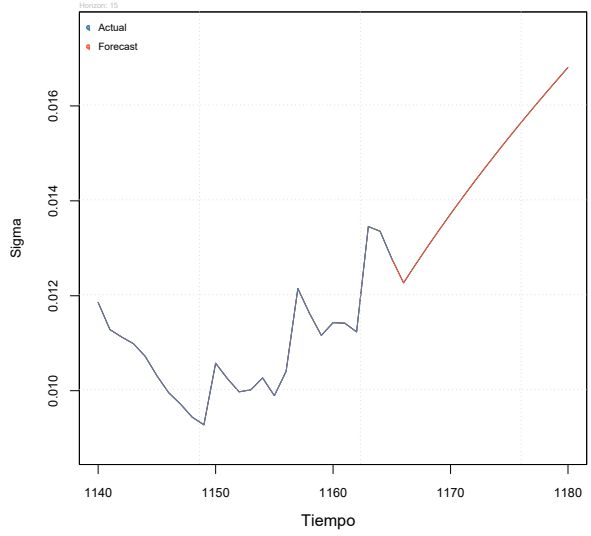

(b) $\operatorname{IGARCH}(1,1)$

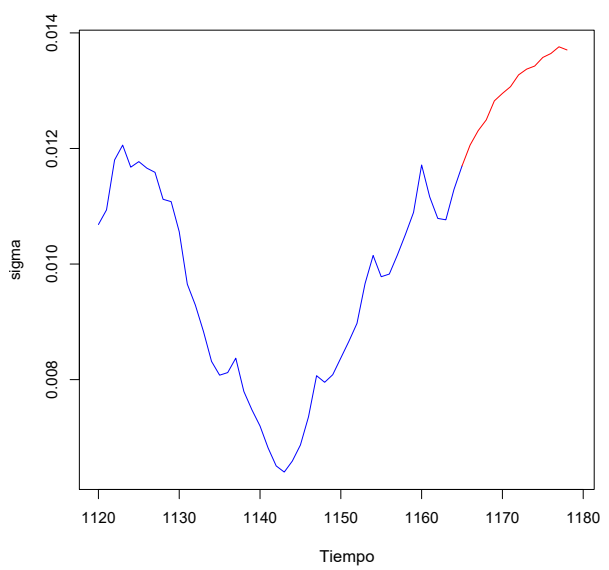

(d) SVt-AR(1)

Fuente: Elaboración propia.

$$
\begin{aligned}
\text { MAPE } & =\frac{1}{15} \sum_{t=1166}^{1181}\left|\frac{X_{t}-\widehat{X_{t}}}{X_{t}}\right|, \\
\text { AMAPE } & =\frac{1}{15} \sum_{t=1166}^{1181}\left|\frac{X_{t}-\widehat{X_{t}}}{X_{t}+\widehat{X_{t}}}\right|,
\end{aligned}
$$

donde MAE es definido como el error absoluto medio, CME el error cuadrático medio, MAPE el error porcentual absoluto medio y AMAPE el error porcentual absoluto medio. 
Tabla 6: Pronósticos precios de cierre CEMARGOS.

\begin{tabular}{|l|l|l|l|l|l|}
\hline \multirow{2}{*}{ Fecha } & Precio & \multicolumn{4}{|c|}{ Precio pronosticado } \\
\cline { 3 - 6 } & & GARCH(1,1) & IGARCH(1,1) & EGARCH(1,1) & SVt-AR(1) \\
\hline \hline $21 / 03 / 2017$ & 11860 & 11993.1395 & 11986.1752 & 11996.4459 & 11979.3693 \\
\hline $22 / 03 / 2017$ & 11880 & 12119.4770 & 12102.3235 & 12124.9351 & 12086.8144 \\
\hline $23 / 03 / 2017$ & 11960 & 11814.5930 & 11823.0092 & 11819.2680 & 11828.1940 \\
\hline $24 / 03 / 2017$ & 11820 & 12038.6706 & 12028.7527 & 12041.2774 & 12017.8682 \\
\hline $27 / 03 / 2017$ & 11920 & 11934.8446 & 11933.3671 & 11939.5198 & 11931.0009 \\
\hline $28 / 03 / 2017$ & 11920 & 11918.4807 & 11918.2540 & 11923.6018 & 11917.2071 \\
\hline $29 / 03 / 2017$ & 11860 & 12019.2199 & 12011.7678 & 12020.8686 & 12001.4441 \\
\hline $30 / 03 / 2017$ & 11960 & 11789.1527 & 11796.8987 & 11800.2781 & 11810.6725 \\
\hline $31 / 03 / 2017$ & 11800 & 11754.9784 & 11764.7173 & 11767.6585 & 11782.1956 \\
\hline $03 / 04 / 2017$ & 11840 & 11739.4142 & 11749.9407 & 11752.8686 & 11769.2840 \\
\hline $04 / 04 / 2017$ & 11860 & 11737.0253 & 11747.6522 & 11750.6074 & 11767.3158 \\
\hline $05 / 04 / 2017$ & 11880 & 11606.5347 & 11621.6346 & 11627.5667 & 11659.7316 \\
\hline $06 / 04 / 2017$ & 11900 & 11761.3398 & 11772.6379 & 11773.0585 & 11787.1002 \\
\hline $07 / 04 / 2017$ & 11780 & 11843.0108 & 11853.0232 & 11849.5490 & 11854.1848 \\
\hline $10 / 04 / 2017$ & 11860 & 12013.3466 & 12022.3934 & 12008.5833 & 11992.3784 \\
\hline \multicolumn{2}{|c|}{ MAE } & 132.0127 & 125.5372 & 127,5650 & 112.0906 \\
\cline { 2 - 5 } CME & 152.2193 & 144.7215 & 147.5396 & 130.1742 \\
\cline { 2 - 5 } MAPE & 0.0111 & 0.0106 & 0.0107 & 0.0094 \\
\cline { 2 - 5 } AMAPE & 0.0056 & 0.0053 & 0.0054 & 0.0047 \\
\hline
\end{tabular}

Fuente: Elaboración propia.

El modelo SVt-AR(1) posee los menores valores MAE, CME, MAPE y AMAPE con relación a los modelos $\operatorname{GARCH}(1,1), \operatorname{IGARCH}(1,1)$ y $\operatorname{EGARCH}(1,1)$, se confirma que dicho modelo, ademas de pronosticar adecuadamente las volatilidades, pronostica con mínimo error los precios de acciones CEMARGOS.

En la Tabla 7 se muestran los intervalos de confianza al $95 \%$ para los pronósticos obtenidos por el modelo SVt-AR(1), el cual se evidencia que los precios reales se encuentran dentro de las bandas.

\section{Conclusiones}

El modelo EGARCH $(1,1)$ es adecuado para ajustar la heterocedasticidad condicional de los retornos de los precios de la acción de Cementos Argos S.A, pues cumple con los supuestos en sus residuales estandarizados y captura adecuadamente los efectos de apalancamiento producidos por las fluctuaciones en el mercado lo cual influye en el alza o las bajas de precios de cierre, respectivamente. De igual forma, el modelo de volatilidad estocástica no resultó adecuado para explicar la volatili- 
Tabla 7: Intervalos de confianza para los pronósticos precios de cierre CEMARGOS dados por el modelo SVt-AR(1)

\begin{tabular}{|l|l|l|l|}
\hline Fecha & \multirow{2}{*}{ Precio } & \multicolumn{2}{|c|}{ Intervalo de confianza del 95\% } \\
\cline { 3 - 4 } & & Limite Inferior & Limite Superior \\
\hline \hline $21 / 03 / 2017$ & 11860 & 9107.704 & 14851.035 \\
\hline $22 / 03 / 2017$ & 11880 & 9214.844 & 14958.785 \\
\hline $23 / 03 / 2017$ & 11960 & 8956.933 & 14699.455 \\
\hline $24 / 03 / 2017$ & 11820 & 9146.095 & 14889.641 \\
\hline $27 / 03 / 2017$ & 11920 & 9059.468 & 14802.534 \\
\hline $28 / 03 / 2017$ & 11920 & 9045.712 & 14788.703 \\
\hline $29 / 03 / 2017$ & 11860 & 9129.717 & 14873.171 \\
\hline $30 / 03 / 2017$ & 11960 & 8939.456 & 14681.889 \\
\hline $31 / 03 / 2017$ & 11800 & 8911.051 & 14653.340 \\
\hline $03 / 04 / 2017$ & 11840 & 8898.172 & 14640.396 \\
\hline $04 / 04 / 2017$ & 11860 & 8896.209 & 14638.423 \\
\hline $05 / 04 / 2017$ & 11880 & 8788.886 & 14530.578 \\
\hline $06 / 04 / 2017$ & 11900 & 8915.944 & 14658.257 \\
\hline $07 / 04 / 2017$ & 11780 & 8982.856 & 14725.513 \\
\hline $10 / 04 / 2017$ & 11860 & 9120.677 & 14864.080 \\
\hline
\end{tabular}

Fuente: Elaboración propia.

dad diaria de los retornos de los precios de la acción de Cementos Argos S.A, pues sus residuales estandarizados no se ajustan correctamente a la distribución normal y $t$-student, respectivamente.

Por otro lado, los modelos GARCH(1,1), IGARCH(1,1), EGARCH(1,1) y SVt$\mathrm{AR}(1)$ pronostican adecuadamente la incertidumbre diaria de los precios de la acción de Cementos Argos S.A. Sin embargo, el modelo SVt-AR(1) resultó ser el mejor modelo para realizar los pronósticos de volatilidades y precios de cierre diario con mínimo error.

Al considerar los criterios de selección AIC, Shibata y Hannan-Quinn, estos se comportaban de manera similar al criterio BIC. Por consiguiente, se tomó el BIC como criterio de selección para los modelo de volatilidad. Ademas, si consideramos variables dummys para los datos inusuales de la serie de retornos $R_{t}$, esto es, para $t=96,625,869,924$, e incluirlas en la ecuación de la media y/o varianza en los modelos de volatilidad $\operatorname{GARCH}(1,1), \operatorname{IGARCH}(1,1)$ y $\operatorname{EGARCH}(1,1)$, solamente en la ecuación de media, dichas variables resultaron significativas a un nivel del $5 \%$. A pesar que dichos modelos poseen menores BIC con relación a los estimados a lo largo de este documento, no lograron capturar completamente los retornos y sus pronósticos no son relativamente significativos respecto a los estimados. Por lo tanto, estos modelos no se consideraron en este trabajo. 
Para finalizar, se debe tener en cuenta que el número de observaciones puede ser un limitante en el ajuste del modelo, tanto condicional como estocástica, e impide una mejor captura de la volatilidad en los retornos de la acción diaria de Cementos Argos. Ademas, el criterio de selección BIC en algunos casos no es adecuado y resulta subjetivo la selección del modelo aproximado para dicho fenómeno.

Recibido: 2017-10-01

Aceptado: 2018-12-11

\section{Referencias}

Argos, C. (2012). Reporte integrado 2012, Cementos Argos SA. Recuperado de http://www.argos.com.co/cemargos.

Argos, C. (2017). Sitio para inversionistas. Recuperado de https://www.argos.co/ir/perfil-corporativo/quienes-somos.

Awartani, Basel MA \& Corradi, V. (2005). Predicting the volatility of the S\&P500 stock index via GARCH models: the role of asymmetries. International Journal of Forecasting, 21(1):167-183.

Bollerslev, T. (1986). Generalized autoregressive conditional heteroskedasticity. Journal of econometrics, 31(3):307-327.

De Colombia, B. d. V. (2011). Bolsa de valores de Colombia. Un país todos los valores.

de Jesús Gutiérrez, Raúl \& Gutiérrez, L. C. (2013). Modelación y predicción de la volatilidad con innovaciones de colas pesadas distribuidas: Evidencia empirica para los petróleos maya y mezcla mexicana de exportación. Paradigma económico, 5(1):67-105.

Engle, R. F. (1982). Autoregressive conditional heteroscedasticity with estimates of the variance of united kingdom inflation. Econometrica: Journal of the Econometric Society, pages 987-1007.

Franco, Pilar Beatriz Álvarez \& Restrepo, D. A. . P. F. O. (2007). Estudio de efectos asimétricos y día de la semana en el índice de volatilidad VIX. Revista Ingenierías Universidad de Medellín, 6(11):125-147.

Ghalanos, A. (2013). Introduction to the rugarch package. $R$ vignette.

González, José Ramón Aragonés \& Blanco, C. (1996). Estimación de la volatidad condicional en el mercado de divisas con modelos de la familia GARCH. Investigaciones europeas de dirección y economía de la empresa, 2(3):43-60.

Jiménez Moscoso, J. A. (2014). Distribuciones de probabilidad alternativas para la gestión de riesgo en mercados financieros. PhD thesis, Universidad de Valencia. 
Kastner, G. (2015). Heavy-tailed innovations in the R package stochvol.

Kastner, G. (2016a). Dealing with stochastic volatility in time series using the R package stochvol. Journal of Statistical Software, 69(5):1-30.

Kastner, G. (2016b). Efficient bayesian inference for stochastic volatility (SV) Models R package stochvol version 1.2.3.

Tsay, R. S. (2014). An introduction to analysis of financial data with R. John Wiley \& Sons. 